myelitis, club-foot, congenital dislocation of the hip, and rheumatoid arthritis, early recognition and prompt treatment minimize the necessary surgery and greatly improve the final results.

1 Wynne-Davies, R., Fournal of Bone and Foint Surgery, 1968, 50b, 24.

2 Bergofsky, E. H., Turino, G. M., and Fishman, A. P., Medicine, 1959, 38, 263.

3 Dwyer, A. F., Fournal of Bone and foint Surgery, 1970, 52B, 782.

Ponseti, I. V., and Friedman, B., Fournal of Bone and foint Surgery, 1950, $32 \mathrm{~A}, 381$.

James, J. I. P., Fournal of Bone and foint Surgery, 1951, 33B, 399.

6 James, J. I. P., Fournal of Bone and Foint Surgery, 1954, 36B, 36.

7 James, J. I. P., Scoliosis. Edinburgh, Livingstone, 1967.

Harrington, P. R., Fournal of Bone and Foint Surgery, 1962, 44A, 591.

O'Brien, J. P., Yau, A. C. M. C., Smith, T. K., and Hodgson, A. R. fournal of Bone and foint Surgery, 1971, $53 \mathbf{B}, 217$.

10 Dwyer, A. F., fournal of Bone and foint Surgery, 1970, 52B, 782.

\section{Safe Prescribing in Liver Disease}

Prescribing for patients with liver disease calls for more than usual caution, because the effects of drugs in this situation are unpredictable. Many drugs are metabolized in microsomal enzyme systems of the liver, but there is no firm evidence that altered drug metabolism invariably accompanies hepatic dysfunction. Indeed a number of studies have shown normal plasma clearance rates for several drugs including phenylbutazone, $^{1}$ acetylsalicylic acid, phenazone, ${ }^{2}$ and chlorpromazine ${ }^{3}$ in patients with cirrhosis. But in other studies both a normal ${ }^{4}$ and depressed ${ }^{5}$ plasma clearance of tolbutamide have been found, and similarly conflicting results have been obtained with thiopentone. ${ }^{6} 7$

It has been suggested that the diseased liver, with a diminished content of hepatocytes, can metabolize drugs at a normal rate because concomitant treatment with other drugs has stimulated the microsomal enzymes in the remaining cells to increased levels of activity. ${ }^{8}$ This process of drugmediated stimulation of hepatic enzymes is known as enzyme induction.

Many drugs have inducing properties, 9 the best known being phenobarbitone, but others which are frequently prescribed to patients with liver diseases, including corticosteroids and spironolactone, are also enzyme inducers. In a recent study, ${ }^{10}$ normal levels of hepatic NADPH cytochrome c reductase, which is a component common to all hepatic enzyme systems for the metabolism of drugs, were found in children with chronic liver disease. However, the rise in this enzyme after administration of phenobarbitone was lower than in normal persons, perhaps because the diminished content of microsomal enzymes had already been stimulated to near maximal activity. In these children there was no history of other treatment by enzyme-inducing drugs, but it is possible that this effect could also be mediated by increases in the plasma levels of certain endogenous compounds with inducing properties, such as steroids, as a result of the liver disease.

A recent study by L. W. Powell and E. Axelsen ${ }^{11}$ is of interest in this connexion and also raises a number of therapeutic questions. These workers determined the degree of conversion of an intravenous dose of prednisone to its metabolically active form, prednisolone. Less than normal conversion was found in patients with active liver disease, whether acute or chronic, but in those whose disease was inactive the drug was metabolized normally. Furthermore, the clearance rate of an intravenously administered dose of prednisolone from the plasma was diminished in patients with active disease. But in this group of patients less of the prednisolone was bound to plasma proteins, this correlating with reduced concentrations of serum albumin. Thus, although there was impaired activation of prednisone to prednisolone by the liver microsomal enzymes, such prednisolone as was formed was more biologically active, firstly because less of it was bound to plasma proteins and secondly because the rate of removal from the plasma was lower. Prednisolone rather than prednisone should therefore be used whenever corticosteroids are indicated in the treatment of active liver disease, but the dose should be carefully regulated because of the increased likelihood of side effects from this drug. Most drugs are inactivated by liver enzymes, a few others activated by them, and almost all are to some extent bound to plasma proteins, so it is unlikely that prednisone is the only drug to be affected in this way by active liver disease.

Azathioprine is also metabolized to the active form in the liver, though it is not known which of the metabolites are responsible for immunosuppression. ${ }^{12}$ This may explain why azathioprine alone is apparently ineffective in controlling the progress of active chronic hepatitis. ${ }^{13}$ Animal experiments have shown that azathioprine can be hepatotoxic both in normal dogs ${ }^{14}$ and even more so in rats with established liver disease produced by carbon tetrachloride. ${ }^{15}$ The evidence for a similar effect in man is less certain. ${ }^{16}$ However, if the drug is used in combination with prednisolone in the treatment of active chronic hepatitis, it is wise to give no more than 75-100 mg of azathioprine daily.

There can be no doubt that patients with hepatic decompensation can readily be precipitated into encephalopathy by even small doses of narcotics and sedatives. ${ }^{17}$ This is probably because their central nervous systems are unduly sensitive to such drugs, rather than because of an exaggerated effect from impaired degradation in the liver. ${ }^{3}$ The confused patient with hepatic encephalopathy is particularly at risk from sedatives because even small doses may precipitate deep coma. In such situations physically restraining the patient, though demanding on the staff, may be safer for the patient. As in any other chronic and disabling illness, patients with chronic liver disease may become depressed by their condition. The physician quite rightly may wish to prescribe psychotropic drugs, and the recent study of $M$. H. Morgan and A. E. Read ${ }^{18}$ gives helpful guidance here. These workers showed that monoamine oxidase inhibitors are particularly apt to precipitate hepatic precoma in patients with cirrhosis, and, if an antidepressant is indicated, then a tricyclic compound such as amitriptyline is safer, but doses should be kept low.

Diuretics are frequently necessary for patients with chronic liver disease to control oedema and ascites, but it must never be forgotten that cirrhotics are liable to develop encephalopathy as a result of rapid alterations in fluid and electrolyte balance. Doses of diuretics should be built up slowly from low levels, and weight loss should never be allowed to exceed $0.5 \mathrm{~kg}$ per day. Adequate potassium supplements are essential if hypokalaemia is to be avoided.

Digoxin may occasionally be needed if heart failure develops from ischaemia or other causes. In one study of alcoholic cirrhotics ${ }^{19}$ its metabolism was found to be normal. If liver function is reasonable there is probably no contrain- 
dication to prescribing this drug in normal doses. However, if there are signs of hepatic decompensation, then normal metabolism of digoxin could not be guaranteed, and the effects of the drug will almost certainly be accentuated by electrolyte disturbances and changes in protein binding.

Finally, unexpected effects may be seen with certain drugs. One such example was provided by the logical use of clofibrate ${ }^{20}$ in primary biliary cirrhosis to control the hypercholesterolaemia and thus the disfiguring xanthomata associated with this condition. However, after its administration there was a rise in serum cholesterol rather than the expected fall, and the skin lesions became worse. Impaired excretion of cholesterol into the bile was suggested as the cause of this paradoxical effect.

In summary, little change in prescribing is necessary when liver disease is inactive, though doses should be kept low, and particular care should be taken with sedative and antidepressant drugs. When active liver disease or signs of hepatic decompensation are present, it is likely-though more studies are urgently needed-that drug metabolism is deranged, and the greatest care indeed should be exercised in prescribing.

1 Burns, J. J., et al., fournal of Pharmacology and Experimental Therapeutics, 1953, 109, 346 .

${ }^{2}$ Brodie, B. B., Burns, J. J., and Weiner, M., Medicina Experimentalis, $1959,1,290$.

3 Maxwell, J. D., et al., Clinical Science, 1972, 43, 143.

4 Nelson, E., American fournal of Medical Science, 1964, 248, 657.

5 Ueda, H., et al., Diabetes, 1963, 12, 414.

- Sessions, J. T., Minkel, H. P., Bullard, J. C., and Ingelfinger, F. J., fournal of Clinical Investigation, 1954, 33,1116.

Dournal of Clinical nvestigation, Antesthesia, 1952, 24, 81

'D Dundee, J. W., British fournal of Anaesthesia, 1952, 24, 81, 275.

10 Conney, A. H., Pharmacological Review, 1967, 19, 317 . 1972, 80, 302.

11 Powell, L. W., and Axelsen, E., Gut, 1972, 13, 690.

12 Bach, J. F., and Dardenne, M., Comptes Rendus des Séances de l'Academie des Sciences (Series D), Paris, 1970, 271, 453

13 Soloway, R. D., et al., Gastroenterology, 1972, 63, 820.

14 Starzl, T. E., et al., Surgery, 1965, 58, 131.

15 Reuber, M. D., Archives of Pathology, 1970, 90, 567.

${ }_{16}$ McKay, I. R., Chan, D., and Robson, G., Australian Annals of Medicine, $1970,19,123$.

17 Laidlaw, I, Read, A. E., and Sherlock, S., Gastroenterology, 1961, 40, 389

18 Morgan, M. H., and Read, A. E., Gut, 1972, 13, 697.

19 Morgus, M. H. and Kapadia, G. G., Gastroenterology, 1964, 47, 517.

20 Schaffner, F., Gastroenterology, 1969, 57, 253.

\section{Chemical Contamination of Stored Blood}

Some twenty years ago the incidence of venous thrombosis at the site of many blood transfusions was shown to have resulted from the leaching of toxic constituents of rubber used in the tubing. ${ }^{1}$ It was not difficult to demonstrate by the greatly diminished incidence of these immediate and obvious local adverse reactions that plastic tubing was safer than rubber, and a recommendation to substitute this in transfusion sets was made. ${ }^{2}$

Now a hypothetical hazard has been suggested as a result of investigations first started by the observations that some pharmacological preparations of isolated tissues were adversely affected when the perfusion fluids were run through certain types of polyvinyl chloride (PVC). ${ }^{34}$

The original observers had suggested that the toxic factors were the organometal stabilizers present in the PVC. However, R. J. Jaeger and R. J. Rubin have provided evidence that the toxic factor may be the plasticizer di-2 ethyl hexyl phthalate (DEHP), which may be present in amounts up to $30 \%$ in flexible PVC. They found that DEHP leached into the fluid used to perfuse rats' livers; it accumulated in these livers and was not apparently metabolized. They went on to show that human blood stored in PVC containers in which this plasticizer was incorporated showed a progressively rising concentration of DEHP, up to $5 \mathrm{mg} / 100 \mathrm{ml}$ after 20 days. Finally they were able to identify DEHP in the tissues of some dead patients who during life had received a transfusion of blood stored in this type of container. As DEHP was not found in all patients who had received it in transfused blood, it is possible that some individuals can metabolize it more readily than others. From the fat of seven people dying suddenly without previous transfusion no DEHP was found.

DEHP is not a substance new to plastic technology. Studies on its possible toxic effects on animals were reported over 25 years ago because of its use in plastics that might lead to the contamination of human food stored in PVC. 56 It could be given in large and repeated doses by mouth to rabbits, rats, and dogs and produced no adverse effects by this route or when given intraperitoneally to rats. The absence of any cumulative effect from repeated doses suggested either that the material was poorly absorbed or that it was rapidly metabolized and excreted. There was very little evidence of conjugation in rats or in two men dosed, only about $5 \%$ of the DEHP being excreted as glucuronide, though some was presumably hydrolysed and excreted as phthalic acid.

But, as these authors pointed out, phthalic acid is a strong acid that should be readily excreted if the ester is hydrolysed. The failure of transfused patients to get rid of the small amount DEHP they receive as a contaminant of blood used in transfusion seems to be matched by the inability of fish to rid themselves of the DEHP they accumulate from the small concentration present in the water of contaminated lakes. Thus levels from 400 up to 3,200 parts per million have been found in some fish, and at this level adverse effects have been reported. ${ }^{7}$ The evidence therefore suggests that esterases capable of hyrolysing DEHP are not widespread in warm or cold-blooded creatures, and this may be yet another somewhat unexpected candidate for inclusion in the ranks of persistent organic chemicals.

The levels found in patients that have been transfused are not nearly as high as those found in the fish, though 270 p.p.m. was found in the fat of one person. It is more surprising to find that much lower levels were detected in the lungs and spleen of some of the patients, suggesting storage in some particulate form in the cells of the reticulendothelial system. Whether the presence of DEHP inside the cells can interfere with the normal function of this important system remains uncertain.

The observation ${ }^{8}$ that three doses of $10 \mathrm{ml}$ DEHP per $\mathrm{kg}$ body weight produced teratogenic effects in rats whereas doses of $5 \mathrm{ml} \mathrm{kg}$ did not is probably of no significance as a reflection of possible hazards to patients receiving blood contaminated with DEHP. However, the probability that it was the plasticizer rather than the stabilizers which were responsible for the adverse effects on two perfused pharmacological preparations gives some cause for further consideration.

There has as yet been no publication confirming the findings of Jaeger and Rubin. PVC has been widely used in transfusion equipment for nearly 20 years without reports of harmful effects attributable to DEHP. Thus panic action should be avoided, but a search for alternative esters suitable for use as plasticizers should be pursued. It seems probable 